\title{
Stage IV Liver Cancer
}

National Cancer Institute

\section{Source}

National Cancer Institute. Stage IV Liver Cancer. NCI Thesaurus. Code C7121.

Stage IV carcinoma of the liver according to the American Joint Committee on Cancer, 6th, 7th, and 8th editions. 